Christian Weber

Das Dilemma des modernen Mannes 
Christian Weber

\section{Das Dilemma des modernen Mannes}

Rollenwidersprüche und das Symptom der Eßstörung

Westdeutscher Verlag 
Die Deutsche Bibliothek - CIP-Einheitsaufnahme

\section{Weber, Christian:}

Das Dilemma des modernen Mannes : Rollenwidersprüche und das Symptom der Eßstörung / Christian Weber. - Opladen ; Wiesbaden : Westdt. Verl., 1998

Alle Rechte vorbehalten

(C) Westdeutscher Verlag GmbH, Opladen/Wiesbaden, 1998

Softcover reprint of the hardcover 1st edition 1998

Der Westdeutsche Verlag ist ein Unternehmen der Bertelsmann Fachinformation GmbH.

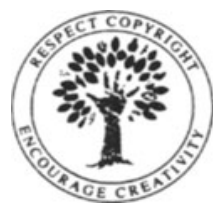

Das Werk einschließlich aller seiner Teile ist urheberrechtlich geschützt. Jede Verwertung außerhalb der engen Grenzen des Urheberrechtsgesetzes ist ohne Zustimmung des Verlags unzulässig und strafbar. Das gilt insbesondere für Vervielfältigungen, Übersetzungen, Mikroverfilmungen und die Einspeicherung und Verarbeitung in elektronischen Systemen.

http://www.westdeutschervlg.de

Höchste inhaltliche und technische Qualität unserer Produkte ist unser Ziel. Bei der Produktion und Verbreitung unserer Bücher wollen wir die Umwelt schonen: Dieses Buch ist auf säurefreiem und chlorfrei gebleichtem Papier gedruckt. Die Einschweißfolie besteht aus Polyäthylen und damit aus organischen Grundstoffen, die weder bei der Herstellung noch bei der Verbrennung Schadstoffe freisetzen.

Umschlaggestaltung: Christine Huth, Wiesbaden 


\section{Danksagung}

Danken will ich zuerst den magersüchtigen und bulimischen Frauen und Männern, denen ich begegnet bin, für die Bereitschaft zur Auseinandersetzung und Diskussion sowie für die Ermöglichung der wissenschaftlichen Untersuchung.

Herzlich zu danken ist Herrn Prof. Dr. Alexander Schuller vom Institut für Soziale Medizin und Medizinische Psychologie der Freien Universität für die Betreuung während der Durchführung meines Dissertationsprojektes sowie vielfältige Hinweise, Anregungen und Kritik. Weiter zu danken ist Herrn Priv.-Doz. Dr. Nikolaus Heim und Frau Dr. Jutta Anna Kleber für ihre Unterstützung und Beratung sowie Herrn Dipl.-Soz. Joachim Koch für die Einführung in die Methodik der objektiven Hermeneutik.

Des weiteren danke ich Stefan Dreßke, Daniela Zippan, Petra Pflugfelder, Marion Mixdorff, Susanne Rompakias und den anderen Teilnehmern am Projekttutorium 1991-1993 "Eßstörungen in Ost und West: Ein Geschichtsaustausch" an der Freien Universität. Harte Diskussionen und gemeinsame Arbeit haben mir Mut gemacht, an meinen Fragestellungen weiterzuarbeiten.

Schließlich danke ich meinen Eltern für ihre Unterstützung.

Christian Weber

Berlin, Februar 1998 


\section{Inhalt}

1. Stand der Forschung: Wissenschaftlicher Diskurs und

Diskurs der Betroffenen ............................................................. 13

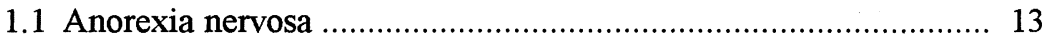

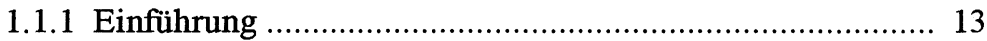

1.1.2 Definitionen und Klassifikationen ................................. 15

1.1.3 Epidemiologie ............................................................. 16

1.1.4 Krankheitsentität Eßstörung:

Anorexie, Eßsucht, Bulimie ........................................ 17

1.1.4.1 Erklärungsmodelle ............................................... 17

1.1.4.2 Einheitlichkeit des Krankheitsbildes Anorexie/ Bulimie: Variante, Syndrom, Entität? ........................... 18

1.1.5 Soziale Figuren des Krankheitsbildes der Anorexia nervosa ........................................................ 19

1.1.5.1 Frauenrolle und Auseinandersetzung mit der Mutter .......................................................... 20

1.1.5.2 Perfektionismus ..................................................... 21

1.1.5.3 Askese: gute und schlechte Speisen .......................... 22

1.1.5.4 Hypermotorik .......................................................... 23

1.1.5.5 Körperbild und Selbstbild:

Dysmorphophobia, falsche Perzeption ......................... 25

1.1.5.6 Heimlichkeit und Schein, Schuld und Scham ............... 26

1.1.6 Exkurs: Anorexie in der Literatur ................................. 27

1.2 Anorexia nervosa masculina ............................................... 28

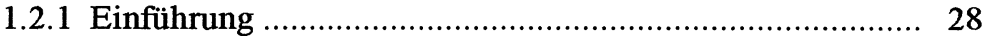

1.2.1.1 Die neuen Männer .................................................... 28

1.2.1.2 Männliche Eßstörung ............................................. 30

1.2.2 Der wissenschaftliche Diskurs zur männlichen Eßstörung ................................................. 31

1.2.2.1 Einzelfallstudien ..................................................... 32

1.2.2.2 Aggregierte Studien mit statistischer Auswertung ......... 35

1.2.2.3 Vergleichsstudien ...................................................... 36

1.2.3 Soziale Figuren des Krankheitsbildes der männlichen Eßstörung .................................................... 40

1.2.3.1 Geschlechtsrollenidentität und Androgynität ................. 40 
1.2.3.2 Homosexualität .................................................... 42

1.2.3.3 Aggressivität, Perversion .............................................. 44

1.2.3.4 Entgrenzung und Psychose ......................................... 45

1.2.4 Exkurs: Männliche Anorexie und Literatur ..................... 47

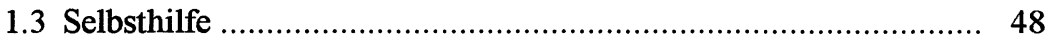

1.3.1 Selbsthilfegruppen .............................................. 48

1.3.1.1 Selbsthilfe als gesellschaftliche Erscheinung in der Bundesrepublik Deutschland der 70er und 80er Jahre ..... 48

1.3.1.2 Selbsthilfegruppen: Setting und Dynamik .................... 50

1.3.2 Selbsthilfe als theoretisches Konzept ............................. 51

1.3.2.1 Selbsthilfe als politisches Konzept ............................. 51

1.3.2.2 Selbsthilfe als ethisches Konzept ............................. 52

1.3.3 Wissenschaftliche Erforschung von Selbsthilfe ................. 53

1.4 Zwischenzusammenfassung .................................................. 54

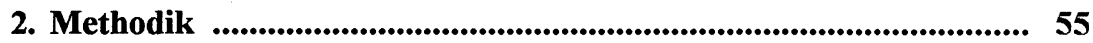

2.1 Die Theorie der Methoden: Zum Feld der Untersuchung und der Bedeutung von Methoden und Technik ......................... 55

2.2 Die angewandten Methoden der Empirie ................................... 57

2.2.1 Angewandte Methoden der Empirie: Interview ............... 57

2.2.2 Angewandte Methoden der Empirie:

Teilnehmende Beobachtung ........................................... 58

2.2.3 Angewandte Methoden der Empirie: Transkription ........... 59

2.2.4 Angewandte Methoden der Empirie: Interpretation .......... 60

3. Begründung und Ablauf der Studie ..........................................63

3.1 Erkenntnisinteresse, Fragestellung ..........................................63 63

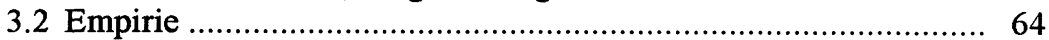

3.3 Diskussion, Interpretation und Auswertung …........................... 65

4. Interpretation des Protokolls eines Interviews mit einem männlichen Eßgestörten und Teilnehmer einer Selbsthilfegruppe

4.1 Interpretation des Protokolls:

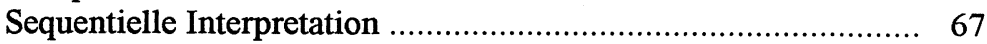

4.1.1 Interpretation dreier Sequenzen ................................... 67

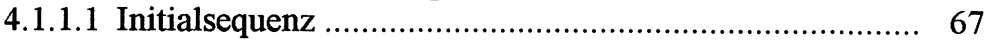


4.1.1.2 Interpretation einer Mittelsequenz ............................ 69

4.1.1.3 Interpretation der Endsequenz .................................. 71

4.1.2 Paraphrase und Übersicht über

Ablauf des Interviews ................................................... 72

4.1.3 Interpretation weiterer inhaltsbezogener Sequenzen .......... 73

4.1.3.1 Sequenzen: Leistungsorientierung, Sicherheit und spontanes Leben .................................................. 73

4.1.3.2 Sequenz: Düstere Szene ........................................... 75

4.1.3.3 Sequenz: Szene der Idylle ........................................ 76

4.2 Interpretation des Gesamtprotokolls ....................................... 77

4.2.1 Kontext, latenter und manifester Gehalt,

Interaktionsbedeutung (Ebenen Oevermann 0-5) ............ 77

4.2.1.1 Kontext ............................................................... 77

4.2.1.2 Wiedergabe der expliziten Äußerung .......................... 77

4.2.1.3 Intention .................................................................. 78

4.2.1.4 Explikation latenter Motive ......................................... 78

4.2.1.5 Explikation in bezug auf Funktion von Verteilung von Interaktionsrollen .............................. 79

4.2.1.6 Interpretation der sprachlichen Merkmale .................. 79

4.2.2 Durchgehende Kommunikationsfiguren, Inhalt und Zusammenfassung (Ebenen Oevermann 6 u. 7) ......... 81

4.2.2.1 Inhalt und Interaktion ................................................ 81

4.2.2.2 Explikation allgemeiner Zusammenhänge und Zusammenfassung

5. Diskussion

5.1 Zur Situierung in Berlin: westliche und östliche

Determinanten .

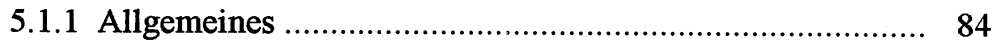

5.1.2 Situierung und Eßstörung ......................................... 85

5.1.3 Situierung und Selbsthilfe ......................................... 87

5.1.4 In dem protokollierten Interview................................... 87

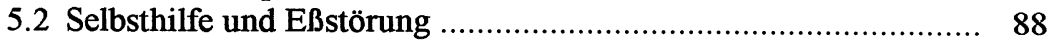

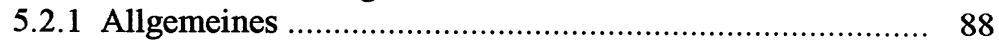

5.2.2 In dem protokollierten Interview ................................ 90

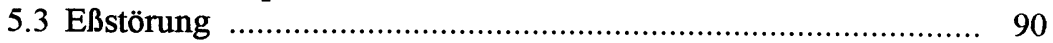

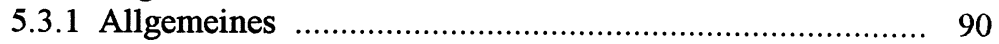

5.3.2 In dem protokollierten Interview ................................. 91 
5.3.3 Eßstörung zwischen sozialer Konstruktion und biologischer Wirklichkeit ........................................... 92

5.4 Männliche Eßstörung und der moderne Mann .......................... 92

5.4.1 Allgemeines ............................................................. 92

5.4.2 In dem protokollierten Interview.................................... 93

$5.5 \mathrm{Zu}$ Methodik und Relevanz ................................................ 95

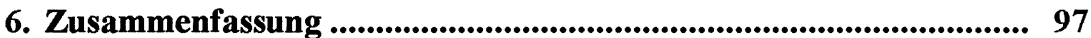

7. Literaturverzeichnis ........................................................... 100

8. Genutzte CD-ROM-Datenbanken .......................................... 122

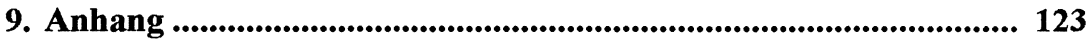

9.1 Tabellarische Übersicht Beratungen der Beratungstelle

DICK und DÜNN in Berlin ................................................. 123

9.2 Tabellarische Übersichten wissenschaftlicher

Studien zu männlicher Eßstörung ........................................ 123

9.2.1 Überwiegend theoretische Studien ohne eigene

Fallpräsentation ....................................................... 123

9.2.2 Einzelfallstudien..................................................... 124

9.2.3 Aggregierte Studien mit statistischer Auswertung ........... 126

9.2.4 Vergleichsstudien .................................................. 127

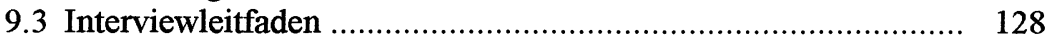

9.4 Transkriptionskonventionen ................................................ 129

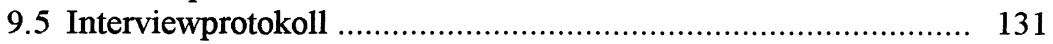

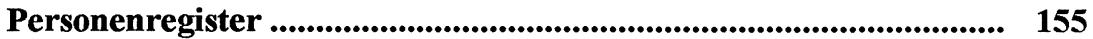

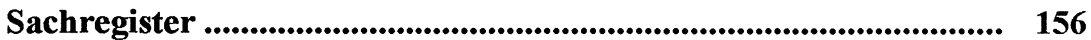

